\title{
Graph Signal Processing: Vertex Multiplication
}

\author{
Bunyamin Kartal, Yigit E. Bayiz, and Aykut Koç ¿ ${ }^{\circledR}$, Senior Member, IEEE
}

\begin{abstract}
On the Euclidean domains of classical signal processing, linking of signal samples to underlying coordinate structures is straightforward. While graph adjacency matrices totally define the quantitative associations among the underlying graph vertices, a major problem in graph signal processing is the lack of explicit association of vertices with an underlying coordinate structure. To make this link, we propose an operation, called the vertex multiplication $(V M)$, which is defined for graphs and can operate on graph signals. VM, which generalizes the coordinate multiplication (CM) operation in time series signals, can be interpreted as an operator that assigns a coordinate structure to a graph. By using the graph domain extension of differentiation and graph Fourier transform (GFT), VM is defined such that it shows Fourier duality that differentiation and CM operations are duals of each other under Fourier transformation (FT). Numerical examples and applications are also presented.
\end{abstract}

Index Terms-Graph signal processing (GSP), graph Fourier transform (GFT), duality, coordinate multiplication, vertex multiplication.

\section{INTRODUCTION}

C LASSICAL digital signal processing (DSP) provides a useful tool for the analysis of signals defined by sampling an Euclidian space such as time-series signals and the $\mathbb{R}^{2}$ plane for images. However, the DSP theory is not designed to capture the complicated structures of large networks, such as social and economic networks, networks arising from the world wide web, and sensor networks. The graph signal processing (GSP) provides a framework that can make the analysis of these networks and processing signals defined on them possible, [1]-[14]. Graph based extensions to machine learning and analysis of brain signals have also been studied [15], [16].

Classical signal processing has been extended to the graph domain [2]-[4]. Specifically, filtering [1]-[6], [13], [14], [17], frequency analysis [5], [8], [18], sampling [10], [19], interpolation [20], Fourier transform (FT) [21], multi-rate signal

Manuscript received December 12, 2020; revised May 29, 2021; accepted May 30, 2021. Date of publication June 3, 2021; date of current version June 29,2021 . The associate editor coordinating the review of this manuscript and approving it for publication was Mr. Yue Gao. (Buyamin Kartal and Yigit E. Bayiz contributed equally to this work.) (Corresponding author: Aykut Koç.)

Bunyamin Kartal is with the Electrical and Electronics Engineering Department, Bilkent University, Ankara 06800, Turkey (e-mail: bunyamin.kartal@ug.bilkent.edu.tr).

Aykut Koç is with the Electrical and Electronics Engineering Department, Bilkent University, Ankara 06800, Turkey, and also with UMRAM, Bilkent University, Ankara 06800, Turkey (e-mail: aykut.koc@bilkent.edu.tr).

Yigit E. Bayiz is with Electrical and Computer Engineering Department, University of Texas at Austin, Austin, TX 78712 USA (e-mail: yigitegeb@gmail.com).

This letter has supplementary downloadable material available at https://doi. org/10.1109/LSP.2021.3086391, provided by the authors.

Digital Object Identifier 10.1109/LSP.2021.3086391 processing [22], signal reconstruction [23], processing of stationary signals and multiscale decomposition methods have been studied for graph signals, [24]-[26].

Much of the developments in the processing of graph signals rely on extending the definition of FT and frequency analysis to graph signals [8]. This allows the translation of many signal processing algorithms to graph signals, and provides a framework that has proved to be foundational in many novel GSP applications, including filtering, sampling and interpolation, [10], [19], [20], big data analysis, [7], and classification, [27], [28]. There are two main approaches for extending the FT to the graph domain. The first is derived from the spectral graph theory and uses the graph Laplacian. This framework describes the graph Fourier transform (GFT) as a change of basis into the basis of the eigenvectors of the graph Laplacian [1]. Despite generally considered to be limited to undirected graphs, it has extensions to directed graphs, [29]. Built on the algebraic signal processing, the second approach is based on the adjacency matrices and describes the GFT as a change of basis into the eigenvectors of the adjacency matrix [14]. This reflects the intuition that the adjacency matrix is analogous to the discrete shift matrix, and the eigenvalues of the latter form a basis for the discrete Fourier transform (DFT). The second approach supports both directed and undirected graphs.

The relationships between operators on graphs and their FT counterparts have also been studied, [1], [2], [5], [8], [14], [18]. Specifically, operations like convolution, translation, modulation, dilation, and filtering as well as the uncertainty relation have all been generalized to GSP domain, [1], [30]. Fourier duality, which is an exact kind of symmetry between the two domains (e.g. translation in one domain is phase multiplication in the other, etc. [1], [31]), is of importance in such generalizations. For example, [32] studied vertex- and spectral-domain convolutions. [33] proposed a dual shift operator that constructs a dual graph in the spectral domain giving it a graph topology. Instead of using classical frequency ordering, [34] proposes a new ordering for the graph Laplacian eigenvectors using the ramified optimal transport theory for better generalizations.

In this letter, by using the Fourier duality for the differentiation operator on graphs, we propose an operation, called the vertex multiplication $(V M)$ for directed graphs. VM mimics the coordinate multiplication (CM) operator of time series signals $(\mathcal{U} f(u)=u f(u))$, which is the Fourier dual of differentiation. While discretization of CM is straightforward, generalization to the graph domain is problematic since the vertices of a graph do not correspond to certain quantitative values, apart from just indices of order. Defined in a matrix form, VM can be interpreted as an operator which assigns a coordinate structure to a graph by associating a "coordinate vector" (represented by the columns of the matrix) for each vertex, and can also operate on graph signals (represented as vectors) through matrix multiplication. Since several established metrics and quantitative manipulations 
can be applied to coordinate vectors, the proposed direct assignment of coordinate structure to the vertex domain can also be instrumental in efforts to define "distance" metrics in the vertex domain, [35], [36], study the notion of smoothness of signals on graphs, [37], localization of signals in the vertex-domain and study of transforms on graphs, [1], [38]-[40].

The proposed VM generalizes a fundamental operation like $\mathrm{CM}$ to directed graph domain and defines the Fourier duality of differentiation for GSP. Moreover, VM, being defined totally consistent with the circulant and dual structure of the DFT, can also be considered a natural way to overcome a major obstacle in embedding the underlying structure of irregular vertex domain to a quantitative coordinate structure assigned to the vertices. This coordinate association is important in the ongoing generalizations from DSP to GSP.

\section{DuAlity RELATION}

The duality between differentiation and CM operators is particularly important in classical signal processing [41], [42]. The duality between time (space) and frequency (spatial-frequency) domains is also one of the most fundamental properties of Fourier analysis. Let $\mathcal{U}, \mathcal{D}$ and $\mathcal{F}$ denote the $\mathrm{CM}$, differentiation and FT operators, respectively. Continuous manifestations of the former two are:

$$
\begin{aligned}
& \mathcal{U} f(u)=u f(u), \\
& \mathcal{D} f(u)=\frac{1}{2 \pi j} \frac{d f(u)}{d u},
\end{aligned}
$$

where $(2 \pi j)^{-1}$ is included to make $\mathcal{U}$ and $\mathcal{D}$ precise Fourier duals (the effect of either in one domain is its dual in the other domain). Then, the duality is given as:

$$
\mathcal{U}=\mathcal{F} \mathcal{D} \mathcal{F}^{-1} \text {. }
$$

Duality can also manifest itself between shift and modulation operations, both of which are fundamental properties of Fourier analysis, [1]. The duality relations in the classical signal processing theory are crucial for understanding much of the underlying theory as well as for being instrumental in applications, [43]. Therefore, extension of these relations to GSP is inescapable. Duality creates a way to define the CM operator without needing coordinates to be explicitly defined once a differential operator on graphs is provided. Therefore, the extension of Fourier duality to graphs can be used to define a coordinate structure on graphs where one replaces $\mathcal{D}$ with the differential operator on graphs and $\mathcal{F}$ with GFT.

\section{GFT AND DIFFERENTIATION ON GRAPHS}

A finite graph $G=(\mathcal{V}, \mathbf{A})$ is a finite set of $N$ ordered points $\mathcal{V}=\left\{v_{0}, v_{1} \ldots v_{N-1}\right\}$ (called vertices) which are connected to each other with some relation. The connections are represented by an adjacency matrix $\mathbf{A}$. The element $a_{i j}$ of $\mathbf{A}$ is the weight of the connection between the $i$ 'th and $j$ 'th vertices, where $i, j=0,1, \ldots, N-1$. In general, connections are directed. For undirected graphs, $\mathbf{A}$ is symmetric with $a_{i j}=a_{j i}$.

Any complex valued function $x$ defined on the set of vertices $\mathcal{V}$, i.e.: $x: \mathcal{V} \rightarrow \mathbb{C}$ is called a graph signal. Since $\mathcal{V}$ is finite, it is convenient to represent $x$ as a vector where each index of $x$ is the value the signal takes on the corresponding vertex:

$$
\mathbf{x}=\left[x_{0}, x_{1}, \ldots, x_{N-1}\right]^{\top}, \quad x_{i}=x\left(v_{i}\right) .
$$
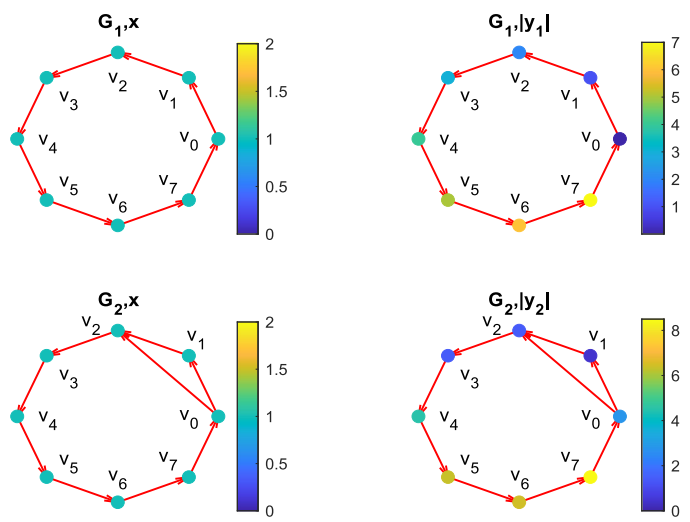

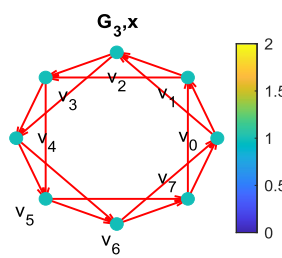

(a)

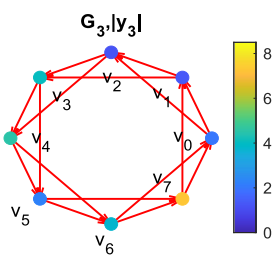

(b)
Fig. 1. Graph structures for Numerical Example 1. (a) The input signal $\mathbf{x}=$ $[1,1, \ldots, 1]$ plotted on vertices with colormaps. (b) Magnitudes of the output signals after $\mathrm{VM}$ operates on the input, i.e., $\mathbf{y}_{\mathbf{i}}=\mathbf{U}_{\mathbf{G}_{\mathbf{i}}} \mathbf{x}$.

When viewed as a vector, operators acting on $x$ can be represented by left multiplication with matrices. Of these operators, the one represented by $\mathbf{A}$ itself is of particular importance, since it implicitly contains the connectivity information of $G$. This operator is called a graph shift and extends the cyclic shift defined on a periodic time series signal in the DSP which has the graph structure $G_{1}$ as shown in Fig. 1(a). In this case, $\mathbf{A}$ is identical to the cyclic shift [8].

Let $G$ be a graph with adjacency matrix $\mathbf{A}$ and $x$ be a graph signal defined on $G$. Then $\mathbf{A}$ can be written in the Jordan canonical form as $\mathbf{A}=\mathbf{V} \mathbf{\Lambda} \mathbf{V}^{-1}$. Then, the Graph Fourier Transform (GFT) of $x$, denoted by $\tilde{x}$, is defined as [14]: $\tilde{x}=\mathbf{V}^{-1} x$. GFT of a signal $x$ is unique up to the ordering of the Jordan blocks in the Jordan canonical form. If $\mathbf{A}$ is diagonalizable, then the Jordan Canonical form is identical to diagonalizing $\mathbf{A}$. In this case GFT becomes a change of basis into the basis of eigenvectors of $\mathbf{A}$, and this process is unique up to the ordering of the Fourier basis vectors. It is also easy to see that if one takes the GFT on a directed circular graph, then the GFT basis becomes the eigenvectors of the cyclic shift matrix, and the GFT reduces to the DFT.

We now consider the definition of differentiation on GSP domain, [44]. Let $x^{c}: \mathbb{R} \rightarrow \mathbb{C}$ be any smooth periodic function with period $T$, and $\mathbf{t}=\left(t_{0}, t_{1}, \ldots, t_{N-1}\right)$ be an ordered set of numbers selected from the interval $[0, T)$. Then an irregular sampling of $x^{c}$ with respect to $\mathbf{t}$ is the finite discrete signal $x \in \mathbb{R}^{N}$ where $x_{n}=x^{c}\left(t_{n}\right)$ for $\forall n \in\{0,1, \ldots, N-1\}$.

Let $S_{0}$ be the circular forward shift operator defined in the space of finite discrete signals of length $N$. Then the matrix representation of $S_{0}$ is of the cyclic shift form. Then, if we let the addition and subtraction on the vector indices to be defined in modulo $N$ (i.e., if we write $x_{-1}=x_{N-1}$ and $t_{-1}=t_{N-1}-T$ ), 
one can use the Taylor series expansion to write:

$$
\begin{aligned}
\left(S_{0} x\right)_{n} & =x_{n-1}=x^{c}\left(t_{n-1}\right)=\sum_{k=0}^{\infty}\left(\left.\frac{\left(t_{n-1}-t_{n}\right)^{k}}{k !} \frac{d^{k}}{d t^{k}}\right|_{t_{n}} x^{c}\right) \\
& =\left(\left(\sum_{k=0}^{\infty} \frac{\left(t_{n-1}-t_{n}\right)^{k}}{k !} \frac{d^{k}}{d t^{k}}\right) x^{c}\right)\left(t_{n}\right) \\
& =\exp \left(\left(t_{n-1}-t_{n}\right) \frac{d}{d t}\right) x^{c}\left(t_{n}\right) .
\end{aligned}
$$

Thus, the matrix manifestations of the discrete differential operator and $S_{0}$ should satisfy $\mathbf{S}_{\mathbf{0}}=\exp \left(-\boldsymbol{\Delta}_{t} \boldsymbol{\nabla}\right)$, where $\boldsymbol{\Delta}_{t}=\operatorname{diag}\left(t_{0}-t_{-1}, t_{1}-t_{0}, \ldots, t_{N-1}-t_{N-2}\right)$. Then, the matrix manifestation of the differential operator defined on unequal sampling of $x^{c}$ resulting in the vector $x$ is defined as:

$$
\boldsymbol{\nabla}=-\boldsymbol{\Delta}_{t}^{-1} \log \mathbf{S}_{\mathbf{0}}
$$

where the complex logarithm can be defined on any branch cut as long as it is consistent throughout the analysis.

\section{GRAPH VERTEX MUltipliCATION}

Consider a graph $G$ with an adjacency matrix $\mathbf{A}$. Then the eigenvalues of $\mathbf{A}$ can be written as the ordered set $\left\{r_{0} e^{j \omega_{0}}, \ldots, r_{N-1} e^{j \omega_{N-1}}\right\}$ where $r_{i}$ 's are the magnitudes and $\omega_{i} \in[0,2 \pi)$ are given in an increasing order. Then for any graph signal $x$ with a GFT of $\tilde{x}$, the elements of $\tilde{x}$ can be ordered with respect to their corresponding eigenvalues. That is, if we write the eigenvalue decomposition of $\mathbf{A}$ as $\mathbf{A}=\mathbf{V} \mathbf{\Lambda} \mathbf{V}^{-1}$ where the diagonal elements of $\boldsymbol{\Lambda}$ are ordered in an increasing order: $\boldsymbol{\Lambda}=\operatorname{diag}\left(r_{0} e^{-j \omega_{0}}, r_{1} e^{-j \omega_{1}}, \ldots, r_{N-1} e^{-j \omega_{N-1}}\right)$. Then, $\tilde{x}=\mathbf{V}^{-1} x$. In this ordering, each coordinate of $\tilde{x}$ are in the order of (possibly irregularly) increasing frequency. Since $\tilde{x}$ has no two coordinates corresponding to the same frequency, we can always find a smooth function $\tilde{x}^{c}: \mathbb{R} \rightarrow \mathbb{C}$ such that $\tilde{x}$ induces an irregular sampling on $\tilde{x}^{c}$ such as: $\tilde{x}_{n}=\tilde{x}^{c}\left(\omega_{n}\right)$. Then the discrete differentiation of $\tilde{x}$ can be defined as:

$$
\left(\nabla_{F} \tilde{x}\right)_{n}
$$

where $\nabla_{F}$ is the FT domain discrete differential operator:

$$
\nabla_{F}=-\Delta_{\omega}^{-1} \log \mathbf{S}_{\mathbf{0}}
$$

where $\quad \boldsymbol{\Delta}_{\omega}=\operatorname{diag}\left(\omega_{0}-\omega_{-1}, \omega_{1}-\omega_{0}, \ldots, \omega_{N-1}-\omega_{N-2}\right)$ with $\omega_{-1}=\left(\omega_{N-1}-2 \pi\right)$. Before proceeding, to be able to use the precise duality relation given in Eq. (3), we first alter the definition for the discrete derivative by dividing Eq. (8) by $j$ :

$$
\tilde{\nabla}_{F}=j \boldsymbol{\Delta}_{\omega}^{-1} \log \mathbf{S}_{\mathbf{0}},
$$

which is analogous to the definition of differential operation with constant multiplier $(2 \pi j)^{-1}$ as given in Eq. (2). (Please note that $2 \pi$ term is already encapsulated in frequency $w$.)

Finally, by using the duality in Eq. (3), we can define the precise Fourier dual of $\tilde{\nabla}_{F}$ as a new operator called vertex multiplication denoted by $\mathcal{U}_{\mathcal{G}}$, in abstract operator notation. The matrix manifestation of $\mathcal{U}_{\mathcal{G}}$, using GFT, is then given by:

$$
\mathbf{U}_{G}=\mathbf{V}^{-\mathbf{1}} \tilde{\nabla}_{F} \mathbf{V}=j \mathbf{V}^{-\mathbf{1}}\left(\boldsymbol{\Delta}_{\omega}^{-1} \log \mathbf{S}_{\mathbf{0}}\right) \mathbf{V}
$$

The proposed VM operator $\mathcal{U}_{\mathcal{G}}$ can be interpreted as a collection of vectors $\mathbf{u}_{\mathbf{i}}$ assigned to each vertex on the graph such that $\mathbf{U}_{G}=\left[\mathbf{u}_{0}, \mathbf{u}_{1}, \ldots, \mathbf{u}_{N-1}\right]$. Also, it operates on a graph signal $\mathbf{x}=\left[x_{0}, \ldots, x_{N-1}\right]^{\top}$ as:

$$
\mathbf{y}=\mathbf{U}_{G} \mathbf{x}=\sum_{i=0}^{N-1} x_{i} \mathbf{u}_{\mathbf{i}} .
$$

This definition provides a generalization of the CM operator. As such, the VM operator computes the superposition of the multiplication of the signal value on each vertex with the vector $\mathbf{u}_{\mathbf{i}}$ associated with the same vertex. Hence, the columns of the VM matrix mimic the coordinate values in the CM operator in DSP (Eq. (1)). Thus, we shall call these columns $\mathbf{u}_{\mathbf{i}}$ the coordinate vector of the i'th vertex. Due to the summation in Eq. (11), the coordinate vector of a vertex has a global effect on the behavior of the VM, and the output graph signal value $\mathbf{y}_{\mathbf{i}}$ depends on values of the input signal at all vertices through the coordinate vectors.

It should be noted that, in our above derivation, we implicitly assume that $\mathbf{A}$ has eigenvalues with distinct arguments (frequencies) when we write the diagonalization of $\mathbf{A}$. This assumption implies that our present framework is valid only for directed graphs. That is because undirected graphs with symmetric adjacency matrices give rise to real eigenvalues with phases being either 0 or $\pi$, which makes ordering of eigenvalues ambiguous. However, our framework that leverages duality is still valid provided that one is able to define a proper differential operator for undirected graphs.

\section{NUMERICAL EXAMPLES}

We first consider the CM of classical DSP and show that it can be interpreted as a special case of VM. The matrix $\mathbf{U}$ that represents the $\mathrm{CM}$ is diagonal. It is composed of one-hot column vectors with non-zero entries existing only at the corresponding indices of each vertex. Then the effect of each coordinate vector in Eq. (11) can be represented locally and thus, the diagonal elements of $\mathbf{U}$ can be assigned to each vertex as proper coordinates. To prove this, consider the graph representation of a time-series as shown by $G_{1}$ in Fig. 1(a). The adjacency matrix in this case is equivalent to the forward time shift, i.e., $\mathbf{A}=\mathbf{S}_{\mathbf{0}}$. The eigenvalues of $\mathbf{A}$ are then equally spaced on the unit circle in the complex plane and are of the form $e^{j \omega_{k}}$ where $\omega_{k}=2 k \pi / N$. Thus we have

$$
\begin{gathered}
\boldsymbol{\Delta}_{\omega}=(1 / N) \operatorname{diag}(2 \pi(0-(-1)), 2 \pi(1-0), \ldots, \\
2 \pi((N-1)-(N-2)))=(2 \pi / N) \mathbf{I},
\end{gathered}
$$

where $\mathbf{I}$ is the $N \times N$ identity matrix. Then, the Fourier domain derivative becomes $-\frac{N}{2 \pi} \log \mathbf{S}_{\mathbf{0}}$. This leads to the following vertex multiplication operator:

$$
\mathbf{U}_{\mathbf{G}}=j \mathbf{V}^{-\mathbf{1}}\left(\frac{N}{2 \pi} \log \mathbf{S}_{\mathbf{0}}\right) \mathbf{V}=j \frac{N}{2 \pi} \log \left(\mathbf{V}^{-\mathbf{1}} \mathbf{S}_{\mathbf{0}} \mathbf{V}\right),
$$

where $\mathbf{V}^{-1}$ reduces to the DFT. Using elementary properties of the DFT, we can obtain

$$
\mathbf{V}^{-1} \mathbf{S}_{\mathbf{0}} \mathbf{V}=\operatorname{diag}\left(e^{-j \omega_{0}}, e^{-j \omega_{1}}, \ldots, e^{-j \omega_{N-1}}\right)=\boldsymbol{\Lambda} .
$$

Then, $\mathbf{U}_{\mathbf{G}}$ is the diagonal matrix with entries $\frac{N \omega_{k}}{2 \pi}$. Finally, $\mathbf{U}_{\mathbf{G}}=\mathbf{U}=\operatorname{diag}(0,1,2, \ldots, N-1)$, which is consistent with the discrete CM operator that can be written as $(\mathbf{U} x)_{n}=n x_{n}$.

Next, we consider our Numerical Example 1 (given in Fig. 1) to show the effects of underlying graphs on the corresponding VM operators and their subsequent effects on a graph signal. 

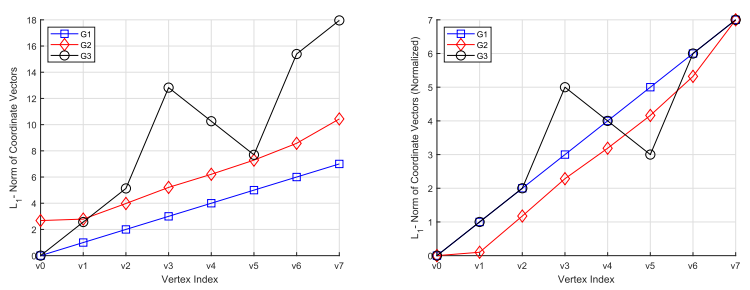

Fig. 2. Coordinates are calculated by $l_{1}$-norm of the coordinate vectors for $G_{1}, G_{2}$, and $G_{3}$. (Left: $l_{1}$-norms. Right: $l_{1}$-norms normalized to the time-series coordinate interval $[0,7]$.

TABLE I

SMOOTHNESS MEASUREMENTS

\begin{tabular}{l|c|c|c|c|}
\hline & $G_{4}$ & $G_{5}$ & $G_{6}$ & $G_{7}$ \\
\hline Total Variation & 1.645 & 1.907 & 2.225 & 2.553 \\
\hline$\left\|\mathbf{u}_{8}\right\|_{1}-\left\|\mathbf{u}_{0}\right\|_{1}$ & 8 & 7.871 & 7.204 & 6.034 \\
\hline
\end{tabular}

$G_{1}$ is the directed circular graph that corresponds to time series signals. We have chosen $G_{2}$ such that it deviates from time series by only having $v_{0}$ makes an extra connection to $v_{2}$; and $G_{3}$ such that each vertex $v_{i}$ has connections to the $v_{i+1}$ and $v_{i+2}$. Fig. 1 also presents the vertex-multiplied output graph signals shown by colormaps on the vertices. It is immediately clear that any deviation from the directed circular graph yields to the matrix manifestation of the VM operator to have complex components. We provide more details on this property in Appendix A. Since VM enables us to define quantitative coordinate vectors, possibilities for manipulations are endless. As an example, in Fig. 2, $l_{1}$-norms of the columns of the VM matrices are plotted, i.e., given $\mathbf{U}_{G}=\left[\mathbf{u}_{0}, \mathbf{u}_{1}, \ldots, \mathbf{u}_{N-1}\right], \mathbf{u}_{i}$ 's are assigned to each vertex to calculate coordinates by $\left\|\mathbf{u}_{i}\right\|_{1}$. Both $l_{1}$-norms and their normalized values to the reference coordinates $(0$, $1, \ldots, 7)$ of the time series by using the scaling $7 \times\left(\left\|\mathbf{u}_{i}\right\|_{1}-\right.$ $\left.\min _{i}\left\|\mathbf{u}_{i}\right\|_{1}\right) /\left(\max _{i}\left\|\mathbf{u}_{i}\right\|_{1}-\min _{i}\left\|\mathbf{u}_{i}\right\|_{1}\right)$ are plotted. Intuitive behavior can be observed in Fig. 2. First, the extra connection between $v_{0}$ and $v_{2}$ at the beginning of the graph almost merges $v_{0}$ and $v_{1}$ so that their coordinates become close to each other; then the linearly increasing coordinate structure continues as in the time series. Second, $G_{3}$ leads to an interesting coordinate pattern due to the regular structure of the graph. A linearly increasing coordinate structure still exists with the abrupt deviation where central vertices $v_{3}$ and $v_{5}$ exchange their coordinates. The reason of this behavior is the dependence of the coordinate ordering of vertices to the ordering of phases of the eigenvalues of the adjacency matrix. We provide the details of this behavior in Appendix B.

As our Numerical Example 2, we apply VM to measure smoothness of signals on graphs. Inspired by the work of [1] that compares smoothness of graph signal $\mathbf{x}=[-0.41,-0.41,-0.35,-0.19,0,0.19,0.35,0.41,0.41]^{T}$ for different graphs by using total variation (TV), we consider four graphs $G_{4}, G_{5}, G_{6}$ and $G_{7}$, each having 9 vertices, as shown in Fig. 3 with increasing TV. TV of a graph signal $\mathrm{x} \in \mathbb{C}^{N}$ defined on a graph with $\mathbf{A} \in \mathbb{C}^{N}$ is defined as [8]: $T V_{G}(\mathbf{x})=\left\|\mathbf{x}-\mathbf{A}^{\text {norm }} \mathbf{x}\right\|$, where $\mathbf{A}^{\text {norm }}=\frac{1}{\left|\lambda_{\max }\right|} \mathbf{A}$ and $\lambda_{\max }$ is the eigenvalue of $\mathbf{A}$ with the largest magnitude. The VM-based smoothness measure is calculated as the difference between the normalized $l_{1}$-norms of the coordinate vectors of the last and the first vertices of each graph. In Table I, TVs of

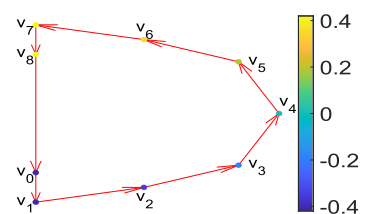

(a) $G_{4}\left(T V_{G}(\mathbf{x})=1.645\right)$

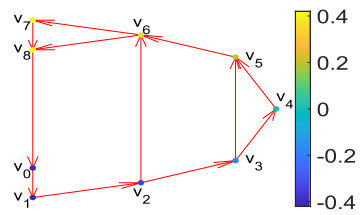

(c) $G_{6}\left(T V_{G}(\mathbf{x})=2.225\right)$

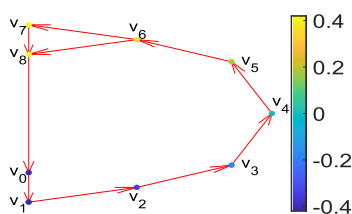

(b) $G_{5}\left(T V_{G}(\mathbf{x})=1.907\right)$

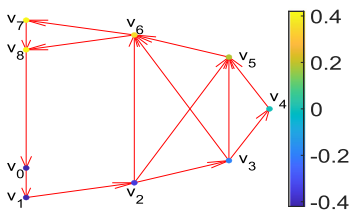

(d) $G_{7}\left(T V_{G}(\mathbf{x})=2.553\right)$
Fig. 3. Example graphs for smoothness measurement.

the signal $\mathrm{x}$ on $G_{4}, G_{5}, G_{6}$ and $G_{7}$ along with the smoothness measure obtained by only the coordinate vectors are shown.

As can be seen in Table I, as one goes from $G_{4}$ to $G_{7}$, the inter-connectivity of the graph structures increases while the graph signals on them are same. This in turn leads to increases in $T V_{G}(s)$ values while the difference between the $l_{1}$-norms of the last and the first coordinate vectors decrease. This result is expected and our measure is in consistence with TV. Vertex coordinates get more and more densely packed due to the increasing number of interconnections. In other words, vertices become "closer" and the variations of the graph signal on this graph structure would be amplified due to this closeness, resulting in higher TVs and reducing smoothness. This gives us a general measure of smoothness of the underlying graph structures irrespective of graph signals that may be defined on them, unlike TV that requires the knowledge of both the graph signal and the graph structure. Above all, being able to represent vertices with theoretically consistent quantitative vectors opens up the path for any kind of mathematical manipulations that involve vectors for a variety of possible future purposes. We believe that VM could open up further directions for research into several other utilizations of the coordinate vectors and the examples such as the above demonstrations can be expanded as future works.

\section{CONCLUSIONS}

Generalization of the CM operation to the graph domain for directed graphs is proposed. The proposed VM is in consistence with the classical signal processing where differentiation and $\mathrm{CM}$ are Fourier duals. VM is an operator that assigns a coordinate structure to a graph by assigning each vertex a coordinate vector. Coordinate vectors, which are intrinsically consistent with the FT theory and its dual structure, can be further manipulated to assign single coordinates to the vertices or for other purposes. Given an adjacency matrix, such an explicit coordinate association is helpful in the ongoing generalizations to GSP. It can also lead to new theoretical and computational endeavors, and deepen our theoretical understanding of the link between vertex and frequency domains with possible insights and applications to the notion of smoothness, distance metrics and localization in the vertex-domain, and transform designs for graph signals. Extension to undirected graphs could be a possible direction for future work. 


\section{REFERENCES}

[1] D. I. Shuman, S. K. Narang, P. Frossard, A. Ortega, and P. Vandergheynst, "The emerging field of signal processing on graphs: Extending highdimensional data analysis to networks and other irregular domains," IEEE Signal Process. Mag., vol. 30, no. 3, pp. 83-98, May 2013.

[2] A. Ortega, P. Frossard, J. Kovačević, J. M. F. Moura, and P. Vandergheynst, "Graph signal processing: Overview, challenges, and applications," Proc. IEEE, vol. 106, no. 5, pp. 808-828, May 2018.

[3] G. Ribeiro and J. Lima, "Graph signal processing in a nutshell," J. Com mun. Inf. Syst., vol. 33, no. 1, pp. 219-233, Jan. 2018

[4] L. Stanković, D. P. Mandic, M. Daković, M. Brajović, B. S. Dees, and T. Constantinides, "Data analytics on graphs Part I: Graphs and spectra on graphs," Found. Trends Mach. Learn., vol. 13, no. 1, pp. 1-157, 2019.

[5] L. Stanković, D. Mandic, M. Daković, M. Brajović, B. Scalzo Dees, and A. Constantinides, "Data analytics on graphs Part II: Signals on graphs," Found. Trends Mach. Learn., vol. 13, nos. 2-3, pp. 158-331, 2019.

[6] A. Gavili and X. Zhang, "On the shift operator, graph frequency, and optimal filtering in graph signal processing," IEEE Trans. Signal Process., vol. 65 , no. 23 , pp. 6303-6318, Dec. 2017.

[7] A. Sandryhaila and J. M. F. Moura, "Big data analysis with signal processing on graphs: Representation and processing of massive data sets with irregular structure," IEEE Signal Process. Mag., vol. 31, no. 5, pp. 80-90, Sep. 2014.

[8] A. Sandryhaila and J. M. F. Moura, "Discrete signal processing on graphs: Frequency analysis," IEEE Trans. Signal Process., vol. 62, no. 12, pp. 3042-3054, Jun. 2014.

[9] L. Stanković et al., "Vertex-frequency graph signal processing: A comprehensive review," Digit. Signal Process., vol. 107, 2020, Art. no. 102802.

[10] S. Chen, R. Varma, A. Sandryhaila, and J. Kovačević, "Discrete signal processing on graphs: Sampling theory," IEEE Trans. Signal Process., vol. 63, no. 24, pp. 6510-6523, Dec. 2015.

[11] Y. Wang, B. Li, and Q. Cheng, "The fractional fourier transform on graphs," in Proc. Asia-Pacific Signal Inf. Process. Assoc. Annu. Summit Conf., Dec. 2017, pp. 105-110.

[12] Y. Wang and B. Li, "The fractional fourier transform on graphs: Sampling and recovery," in Proc. 14th IEEE Int. Conf. Signal Process., , 2018, pp. 1103-1108.

[13] A. Sandryhaila and J. M. F. Moura, "Discrete signal processing on graphs: Graph filters," in Proc. IEEE Int. Conf. Acoust., Speech Signal Process., May 2013, pp. 6163-6166.

[14] A. Sandryhaila and J. M. F. Moura, "Discrete signal processing on graphs," IEEE Trans. Signal Process., vol. 61, no. 7, pp. 1644-1656, Apr. 2013

[15] L. Stanković et al., "Data analytics on graphs Part III: Machine learning on graphs, from graph topology to applications," Found. Trends Mach. Learn., vol. 13, no. 4, pp. 332-530, 2020.

[16] W. Huang, L. Goldsberry, N. F. Wymbs, S. T. Grafton, D. S. Bassett, and A. Ribeiro, "Graph frequency analysis of brain signals," IEEE J. Sel. Topics Signal Process., vol. 10, no. 7, pp. 1189-1203, Oct. 2016.

[17] F. Hua, C. Richard, J. Chen, H. Wang, P. Borgnat, and P. Gonçalves, "Learning combination of graph filters for graph signal modeling," IEEE Signal Process. Lett., vol. 26, no. 12, pp. 1912-1916, Dec. 2019.

[18] F. Grassi, A. Loukas, N. Perraudin, and B. Ricaud, "A time-vertex signal processing framework: Scalable processing and meaningful representations for time-series on graphs," IEEE Trans. Signal Process., vol. 66, no. 3, pp. 817-829, Feb. 2018.

[19] A. Anis, A. Gadde, and A. Ortega, "Towards a sampling theorem for signals on arbitrary graphs," in Proc. IEEE Int. Conf. Acoust., Speech Signal Process., 2014, pp. 3864-3868.

[20] S. K. Narang, A. Gadde, and A. Ortega, "Signal processing techniques for interpolation in graph structured data," in Proc. IEEE Int. Conf. Acoust., Speech Signal Process., 2013, pp. 5445-5449.

[21] A. Sandryhaila and J. M. F. Moura, "Discrete signal processing on graphs: Graph fourier transform," in Proc. IEEE Int. Conf. Acoust., Speech Signal Process., 2013, pp. 6167-6170.

[22] O. Teke and P. P. Vaidyanathan, "Extending classical multirate signal processing theory to graphs-part i: Fundamentals," IEEE Trans. Signal Process., vol. 65, no. 2, pp. 409-422, Jan. 2017.
[23] E. Brugnoli, E. Toscano, and C. Vetro, "Iterative reconstruction of signals on graph," IEEE Signal Process. Lett., vol. 27, pp. 76-80, 2020.

[24] N. Perraudin and P. Vandergheynst, "Stationary signal processing on graphs," IEEE Trans. Signal Process., vol. 65, no. 13, pp. 3462-3477, Jul. 2017.

[25] A. G. Marques, S. Segarra, G. Leus, and A. Ribeiro, "Stationary graph processes and spectral estimation," IEEE Trans. Signal Process., vol. 65, no. 22, pp. 5911-5926, Nov. 2017.

[26] X. Zheng, Y. Y. Tang, J. Pan, and J. Zhou, "Adaptive multiscale decomposition of graph signals," IEEE Signal Process. Lett., vol. 23, no. 10, pp. 1389-1393, Oct. 2016.

[27] S. Chen, F. Cerda, P. Rizzo, J. Bielak, J. H. Garrett, and J. Kovačević, "Semi-supervised multiresolution classification using adaptive graph filtering with application to indirect bridge structural health monitoring," IEEE Trans. Signal Process., vol. 62, no. 11, pp. 2879-2893, Jun. 2014.

[28] S. Chen, A. Sandryhaila, J. M. Moura, and J. Kovačević, "Adaptive graph filtering: Multiresolution classification on graphs," in Proc. IEEE Glob. Conf. Signal Inf. Process., 2013, pp. 427-430.

[29] R. Singh, A. Chakraborty, and B. S. Manoj, "Graph fourier transform based on directed Laplacian," in Proc. Int. Conf. Signal Process. Commun., 2016, pp. 1-5.

[30] A. Agaskar and Y. M. Lu, "Uncertainty principles for signals defined on graphs: Bounds and characterizations," in Proc. IEEE Int. Conf. Acoust., Speech Signal Process., 2012, pp. 3493-3496.

[31] H. M. Ozaktas, Z. Zalevsky, and M. A. Kutay, The Fractional Fourier Transform With Applications in Optics and Signal Processing. New York, NY, USA: Wiley, 2001.

[32] M. Cheung, J. Shi, O. Wright, L. Y. Jiang, X. Liu, and J. M. F. Moura "Graph signal processing and deep learning: Convolution, pooling, and topology," IEEE Signal Process. Mag., vol. 37, no. 6, pp. 139-149, Nov. 2020.

[33] G. Leus, S. Segarra, A. Ribeiro, and A. G. Marques, "The dual graph shift operator: Identifying the support of the frequency domain," J. Fourier Anal. Appl., vol. 27, no. 49, 2021.

[34] N. Saito, "How can we naturally order and organize graph Laplacian eigenvectors?" in Proc. IEEE Stat. Signal Process. Workshop, 2018, pp. 483-487.

[35] D. Ron, I. Safro, and A. Brandt, "Relaxation-based coarsening and multiscale graph organization," Multiscale Model. Simul., vol. 9, no. 1, pp. 407-423, 2011.

[36] S. Lafon and A. B. Lee, "Diffusion maps and coarse-graining: A unified framework for dimensionality reduction, graph partitioning, and data set parameterization," IEEE Trans. Pattern Anal. Mach. Intell., vol. 28, no. 9, pp. 1393-1403, Sep. 2006.

[37] X. Zhu and M. Rabbat, "Approximating signals supported on graphs," in Proc. IEEE Int. Conf. Acoust., Speech Signal Process., 2012, pp. 3921-3924.

[38] R. R. Coifman and M. Maggioni, "Diffusion wavelets," Appl. Comput. Harmon. Anal., vol. 21, no. 1, pp. 53-94, 2006.

[39] D. K. Hammond, P. Vandergheynst, and R. Gribonval, "Wavelets on graphs via spectral graph theory," Appl. Comput. Harmon. Anal., vol. 30, no. 2, pp. 129-150, 2011.

[40] S. K. Narang and A. Ortega, "Perfect reconstruction two-channel wavelet filter banks for graph structured data," IEEE Trans. Signal Process., vol. 60, no. 6, pp. 2786-2799, Jun. 2012.

[41] K. A. Atkinson, Signal Analysis. New York: McGraw-Hill, 1977.

[42] C. Cohen-Tannoudji, B. Diu, and F. Laloe, Quantum Mechanics. New York: Wiley, 1979.

[43] A. Koç, B. Bartan, and H. M. Ozaktas, "Discrete linear canonical transform based on hyperdifferential operators," IEEE Trans. Signal Process., vol. 67, no. 9, pp. 2237-2248, May 2019.

[44] B. S. Dees, L. Stanković, M. Daković, A. G. Constantinides, and D. P. Mandic, "Unitary shift operators on a graph," 2019, arXiv:1909.05767. 